and the second to survive. Only one previous fetus has survived a pregnancy complicated by HSE and the mother, treated with idoxuridine, died 5 days postpartum. Acyclovir reduces mortality of neonatal HSE and is well tolerated but morbidity in surviving infants is high especially if treatment is delayed. Early diagnosis is facilitated by MRI. (Scroth G et al. Neurology 1987:37:179). For a review of the natural history of HSV infection of mother and newborn and therapy of neonatal HSV infection, see Whitley RJ et al. and Infectious Disease Collaboration Antiviral Study Group. Pediatrics 1980: $66: 489-501$.

\title{
HERPES ZOSTER OPHTHALMICUS
}

A 17-month-old boy with $\mathrm{HZO}$ and delayed contralateral hemiparesis following intrauterine varicella exposure is reported from the Dept of Neurology, Univ Texas Med Sch, Houston, TX. He presented with ataxia and a progressive right-sided weakness. His mother had chicken-pox at 8 months of gestation but he appeared normal at birth. A vesicular rash developed 4 weeks before examination in the distribution of the ophthalmic and mandibular divisions of the left trigeminal nerve. CSF showed mononuclear pleocytosis, CT demonstrated multiple areas of hypodensity in the left basal ganglia, and angiography revealed occlusion of the left lenticulostriate arteries. Treatment with Acyclovir for 10 days was followed by recovery except for minimal right hemiparesis. (Leis AA, Butler IJ. Infantile herpes zoster ophthalmicus and acute hemiparesis following intrauterine chickenpox. Neurology 1987:37:1537-1538). Passive immunization of susceptible women exposed to varicella is recommended to reduce the risks of maternal and fetal varicella. The determination of varicella zoster virus membrane antigen or equivalent anti-varicella antibody status in pregnant women exposed to varicella is a rapid, satisfactory method for determining who should receive varicella immunoglobulin passive immunization (MCGregor JA et al. Am J Obstet Gynecol 1987: 157:281).

COMPENT: The authors cited only one similar previous case in a child, a 7-year-old boy. Delayed focal cerebral angiitis and infarction may occur after an interval of days to months between HzO and neurologic complications in adults. Passive immunization of exposed susceptible women reduces risks of maternal and fetal varicella. (MCGregor JA et al. Am J Obstet Gynec 1987:157:281).

\section{LANGUAGE AND BEHAVIOR}

\section{CROSSED APHASIA}

A case of crossed aphasia with persistent language disturbances in a right-handed boy aged 5 yr 9 mos is reported from the Centre Hospitalier Universitaire Vaudois, Lausanne, France. An acute left hemiplegia resulted from occlusion of the internal carotid siphon of undermined cause and demonstrated by arteriography. The boy was mute, his auditory comprehension impaired, and tongue and facial movements apraxic. His first intelligible words (maman and non) were pronounced at 2 months after the onset. The language remained agrammatic and the vocabulary and comprehension poor but the tongue apraxia resolved. Twelve years later, language disturbances were still present although his IQ on the WAIS was 100 full scale, 86 verbal (information 6, comprehension 6, digit memory 5, 